\title{
SPACECRAFT ENERGY STORAGE SYSTEMS
}

\author{
SatCon Technology Corporation \\ 6245 E. Broadway Blvd., Suite 350 \\ Tucson, AZ 85711
}

\begin{abstract}
Wilf Robinson
James Hanks

Len Spina
\end{abstract}

\author{
Doug Havenhill \\ Gary Gisler \\ Dr. Steve Ginter
}

Dr. Sharon Brault

\begin{abstract}
Flywheel Energy Storage Systems represent an exciting alternative to traditional battery storage systems used to power satellites during periods of eclipse. The increasing demand for reliable communication and data access is driving explosive growth in the number of satellite systems being developed as well as their performance requirements. Power on orbit is the key to this performance, and batteries are becoming increasingly unattractive as an energy storage media. Flywheel systems offer very attractive characteristics for both energy storage in terms of energy density and the number of charge/discharge cycles, together with the important side benefit of spacecraft attitude control.
\end{abstract}

\section{$\underline{\text { Introduction }}$}

Operational spacecraft experience periods of solar eclipse which negates the ability of the solar arrays to provide power. An on-board energy storage system is required to maintain spacecraft function during these eclipse periods. Batteries are the traditional method of providing energy storage and have performed this function adequately. Recently, however, projected power requirements for spacecraft have increased. For instance, current communications satellites require $4-6 \mathrm{~kW}$-hr of energy storage resulting in battery systems that account for approximately $12 \%$ of the launch weight. Near term projections show energy storage requirements increasing to 20 $30 \mathrm{~kW}$-hr. If today's batteries with energy densities at the system level of $5-20 \mathrm{Whr} / \mathrm{kg}$ were used to provide this energy storage, batteries would account for $25 \%$ of the launch weight. Additionally advanced batteries have a number of drawbacks including

- Narrow operational temperature range

- Capacity fade over life

- Difficulty in obtaining charge status

- Environmental concerns

- Energy storage and power capacity are coupled. Clearly, alternative energy storage devices require investigation.
One alternative to batteries is flywheel energy storage. The concept for spacecraft flywheel energy storage systems originated in the early 1970's. A number of studies concluded that technological constraints in the areas of power control electronics, magnetic bearings, and composite materials limited the potential benefits of this form of energy storage. However, twenty five years later, significant advances in these key technologies have spurred a new interest in flywheel energy storage. Potential advantages of flywheels include:

- Operation over a wide temperature range

- No capacity fade over life

- Since mechanical energy is proportional to the square of the flywheel speed, the stored energy level is a simple speed measurement

- Environment friendly

- Decoupled energy storage and energy delivery functions allowing large peak power draws without significant increase in weight.

- Energy densities of up to $60 \mathrm{Whr} / \mathrm{kg}$

- Spacecraft Attitude Control.

This paper describes a generic spacecraft flywheel energy storage system currently being developed by SatCon Technology Corporation, generally to fulfill the basic requirements shown in Table 1 . 
Table 1: General Requirements for a Flywheel Energy Storage System

\begin{tabular}{|l|c|c|c|}
\hline \multicolumn{1}{|c|}{ Parameter } & Units & GEO Orbit & $\begin{array}{c}\text { LEO } \\
\text { Orbit }\end{array}$ \\
\hline $\begin{array}{l}\text { Useable energy } \\
\text { (on-orbit) }\end{array}$ & $\mathrm{kW}$-hr & 12 & 6 \\
\hline $\begin{array}{l}\text { Energy density } \\
\text { (goal) }\end{array}$ & Whr/kg & 60 & 20 \\
\hline $\begin{array}{l}\text { Average power } \\
\text { (on-orbit) }\end{array}$ & $\mathrm{kW}$ & 10 & 5 \\
\hline $\begin{array}{l}\text { Bus voltage } \\
\text { Charge/ Discharge }\end{array}$ & cycles & 4,000 & 50,000 \\
\hline Discharge efficiency & $\%$ & 94.6 & 94.6 \\
\hline $\begin{array}{l}\text { Operating } \\
\text { Environment }\end{array}$ & $\%$ & 100 & 28 to 150 \\
\hline Temperature & ${ }^{\circ} \mathrm{C}$ & -18 to +60 & -18 to +60 \\
\hline $\begin{array}{l}\text { Radiation (total } \\
\text { dose) }\end{array}$ & rads & $10^{4}$ & $10^{\circ}$ to $10^{\circ}$ \\
\hline Life & & & 10 \\
\hline Mission life & yrs & 10 & 10 \\
\hline $\begin{array}{l}\text { Mission } \\
\text { Reliability }\end{array}$ & & 0.85 & 0.85 \\
\hline
\end{tabular}

\section{Flywheel Energy Storage System}

A typical flywheel energy storage system shown in Figure 1 consists of a rotating flywheel assembly and a set of control electronics. The energy storage medium is a high speed rotating mass contained in the flywheel assembly, each assembly consists of

- a high speed rotor, which stores the energy

- magnetic bearings for unlimited life, minimizing frictional losses and emitted vibrations

- mechanical touchdown bearings to support launch loads and protect against magnetic bearing anomalies

- a motor/generator to convert electrical energy to mechanical energy and vice versa

- a housing to provide structural support to the bearings and a vacuum enclosure for ground testing.

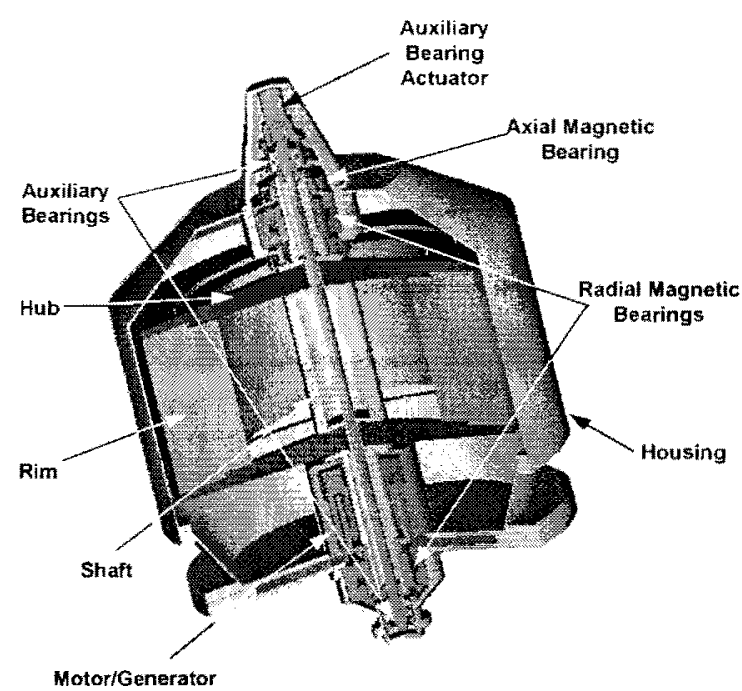

Figure 1: Flywheel Assembly

The power control electronics control the rate at which the energy is extracted or delivered. The primary electronics assemblies within the power control electronics are

- motor/generator power electronics to conduct current into and out of the motor windings

- a digital signal processor providing voltage regulation, motor winding current control, magnetic bearing control, health monitoring, and serial communication for spacecraft command and telemetry

- a secondary power supply to furnish low voltage power for the electronics

- magnetic bearing drive amplifiers for driving the magnetic bearing coils

- an EMI/EMC filter to limit conducted electromagnetic interference to the spacecraft power bus

- auxiliary bearing actuator electronics

A diagram showing the interconnection of the electronic assemblies and the flywheel assembly is shown in Figure 2. 


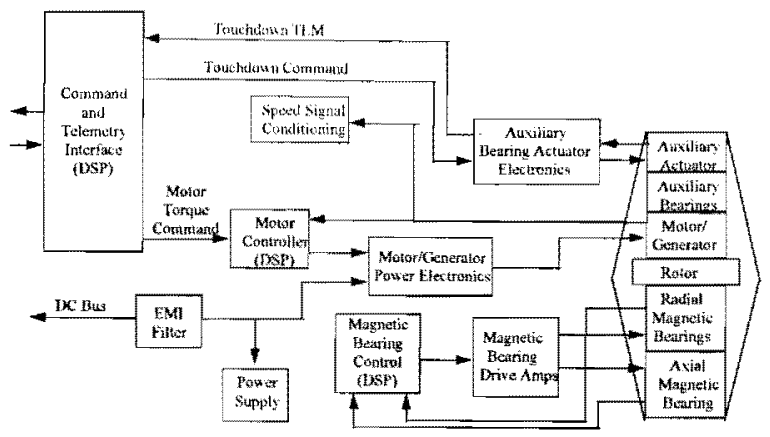

Figure 2: Functional Block Diagram of Flywheel and Electronics

\section{Components}

Technology advancements today in the areas of high strength composites enabling high flywheel speeds (up to $50,000 \mathrm{rpm}$ ), precision magnetic bearings providing high speed rotor support with low friction and no wear, and efficient high voltage power control electronics have made possible practical flywheel energy storage systems.

\section{Rotor}

The high speed rotor used in flywheel energy systems, consists primarily of at least two elements: a central shaft carrying the rotating elements of the magnetic bearings and motor/generator and the inertia rim where the energy is stored. This rim can either be attached directly to the shaft or as a circular annulus attached to the shaft through a hub. See Figures 3 and 4 . Both configurations use a composite of high strength fibers and epoxy to provide the high speed capability, actual selection is generally driven by performance requirements, cost and producibility.

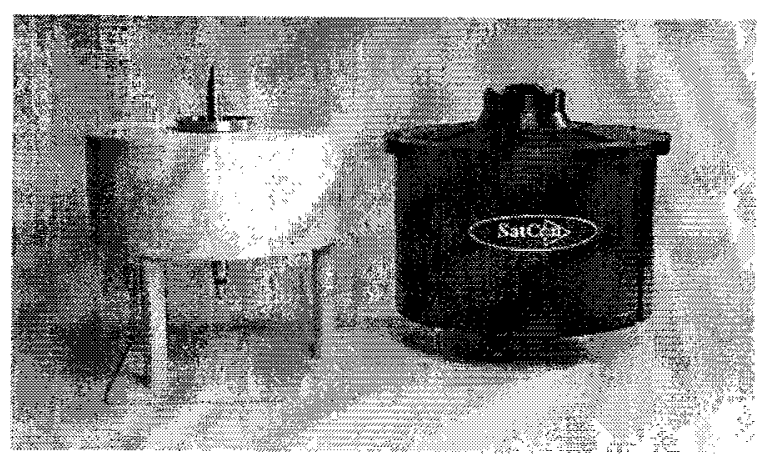

Figure 3

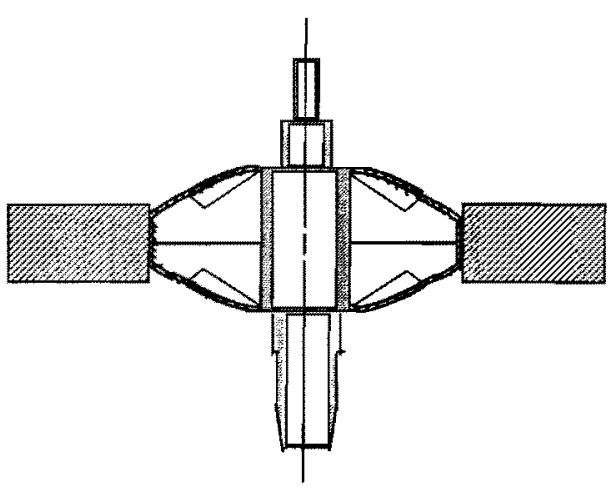

Figure 4

The high speed regime in which these devices operate raises challenging structural, dynamic and safety issues. Design considerations such as mean stresses arising principally from spin speed, cyclic and alternating stress from speed cycles, random vibration, and gyroscopic loads have to be addressed. The radial growth that occurs at these high speeds has been accommodated by using a layered construction in the Figure 3 configuration and tailoring hub flexibility in the rim approach of Figure 4.

Given the relative immaturity of this technology, when compared with all metal rotors, intense efforts are on-going to ensure that material properties are correct, that fatigue and strength meet design expectations and that the fabrication process is verified through actual piece part testing. The derating factors used through the design process are items of constant debate. Process control and the detection of flaws are an on-going major activity.

\section{Magnetic Bearings}

The magnetic bearing is the primary suspension for the high speed rotor. The function of the bearing is to permit the rotor to freely spin about its axial axis, with minimal resistance to the rotational motion. In addition, the bearing must adequately manage rotor displacements in the five remaining degrees of freedom (three translations and two radial rotations) to prevent physical contact of the rotor with the touchdown bearing. Disturbances that result in rotor displacement originate from rotor mass imbalance, the spin motor/generator, acceleration and rotation of the unit mounting base, and force imbalance amongst the actuators themselves. To maximize the bearing forceto-weight ratio an active magnetic bearing system is needed. The total bearing system would be configured with two radial bearings at each end of the rotor 
shaft and one thrust (axial) bearing (Figure 1). Each radial bearing would be comprised of four distinct electromagnetic actuators, each actuator having a stator element, mounted to the unit housing, and an armature element, mounted to the rotor. The stators typically have a "C" cross-sectional geometry with a wire coil wound around each of the two pole faces. The four stators in each radial bearing share a common armature element on the rotor. The thrust bearing is comprised of two electromagnetic actuators. The stator elements typically have a "cup core" crosssectional geometry, with a wire coil bonded into the cup. The two thrust bearing actuators can either share a common armature mounted to the rotor or utilize separate armatures.

When energized with a coil current, the magnetic actuator develops a non-contacting, attractive magnetic force between the stator and armature elements. The force applied to the stator is ultimately reacted onto the satellite via the housing, while the force applied to the rotor pulls it toward the stator. Thus, the actuators are configured as opposing pairs to balance the forces on the rotor. The force developed by the actuator also depends on the "air gap" between the stator pole face and the armature. The force dependence on gap couples the force and rotor motion in a manner that produces unstable rotor dynamics. To achieve a stable magnetic bearing, therefore, requires the use of active feedback control. The force behavior of the magnetic actuator is also highly nonlinear. The actuator force is related to the field flux density via a square law, and the flux density is related to the electromagnet coil current through the nonlinear B-H characteristic of the magnet material, which also exhibits significant hysteresis and saturation. Thus, feedback control is also necessary to linearize and improve the actuator's force performance.

The active controls in the bearing consist of high bandwidth (hundreds of hertz) "inner" feedback loops on each of the actuators and lower bandwidth (10 to 30 hertz) "outer" feedback loops to manage rotor position. This inner-outer loop architecture is illustrated in Figure 5. The inner-outer loop control architecture is a solid approach that works well, provided that the inner actuator control loops perform as required. Experience with magnetic suspensions indicates the best performance is achieved when the inner loops are configured as force command loops, precision force sensors. Alternatively, measurement of the actuator flux density $\beta$ (readily obtained from a Hall effect device) as the primary feedback signal, illustrated in Figure 6, achieves very acceptable force performance for this type of application. In addition, if a sense coil is also employed to provide an additional high frequency feedback signal, then a further marked improvement in the force performance is obtained through a significant increase in loop bandwidth. The sense coil is easily wound with the electromagnet drive coil and provides a measurement producing a magnetic bearing that applies the commanded forces to the rotor. The outer position loops then function to control rotor position by commanding appropriate forces to the inner actuator loops. Thus, the inner loops must function to produce the forces commanded over the frequency range required.

However, controlling forces over a wide bandwidth is quite difficult due to the dynamic behavior of most that is proportional to the time rate flux change $d \phi / d t$ through the sense coil. The use of a Hall device and sense coil for feedback produces a very tight, high bandwidth inner control loop on the electromagnet that gives excellent bearing force performance. A second advantage is that the actuator loop is the major agent in mitigating the inherent instability in the bearing. This situation relieves the high bandwidth requirement on the outer position control loops.

There are five rotor position control loops that manage the three rotor translational and two radial rotational degrees of freedom. These loops are based on measurements of rotor position obtained from noncontacting position transducers. Two types of transducers that have been successfully employed are capacitive based and eddy current based devices. The rotor position loops, in particular the loop bandwidth, establish the effective stiffness of the bearing. The bandwidth in these loops are constrained by digital sampling effects, rotor and housing structural resonances, and the desire to minimize emitted disturbances from the flywheel energy unit. Past experience has shown that loop bandwidths from 10 to 30 $\mathrm{Hz}$ are quite adequate in achieving acceptable bearing behavior. 


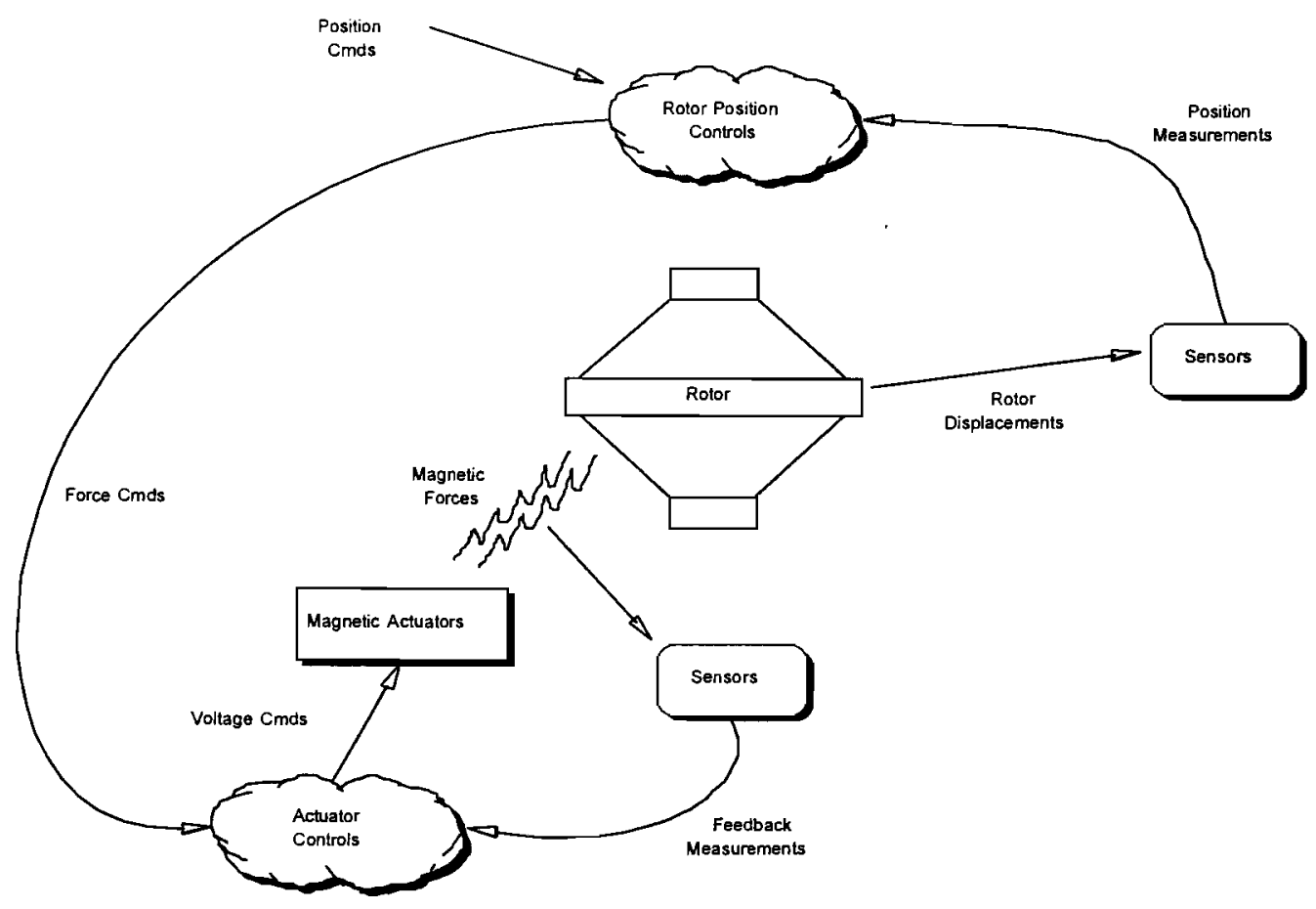

Figure 5: Inner Outer Loop Magnetic Bearing Control Architecture

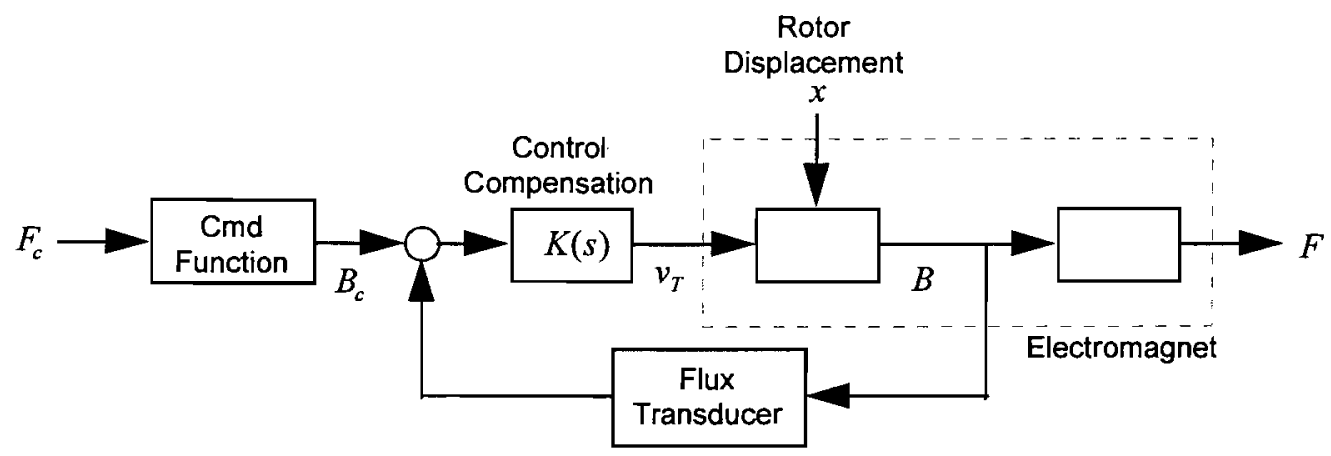

Figure 6: Actuator Control Loop

Magnetic bearings do exhibit magnetic losses that result in a reduction of power efficiency in the flywheel energy storage unit and also generate heat. Thus, attention must be given in the design of the bearing to minimizing the losses. The magnetic losses originate from B-H loop hysteresis, eddy currents in the stator, and foucault currents in the armature. All these losses are due to a magnetic field that changes with time. The eddy and foucault currents are electrical currents induced in the magnet iron when the field changes. These currents themselves tend to set up a magnetic field that opposes the originating change in the magnetic field. In the stator, the eddy currents cause more drive coil current to be used to effect a desired change in the actuator force, leading to a reduction in force efficiency from the bearing. In the armature the foucault currents act like an increase in reluctance that also reduces the bearing force efficiency. Resistive effects in the magnet iron cause the eddy and foucault currents to generate heat which must be addressed in the bearing design. Eddy and foucault currents are principally dealt with by laminating the stator and armature elements and using low resistive metal alloys to the greatest extent possible. 


\section{Auxiliary Bearing System}

The magnetic bearings do not provide the entire rotor suspension function. In particular, the magnetic bearings are not size/weight effective for ground transport and launch. Furthermore, anomalous event on orbit such as power outage or single event upsets can be troublesome to magnetic bearing operation. Thus, a mechanical auxiliary bearing is necessary. The auxiliary bearing does permit high speed, nondestructive contact of rotor with stationary elements on the housing.

This system principally comprises two sets of duplex bearings connected via a rod through the rotor shaft. Two conical surfaces form the connection between ground and the spinning rotor. In normal operation mechanical gaps exist at these conical surfaces, however, should an anomaly occur it is sensed by the onboard health monitors and the auxiliary bearing system actuator is energized closing the gap at the conical surfaces, capturing the rotor and transferring its rotation from the magnetic bearings to the duplex bearings. The high rotation speeds and speed at which capture has to occur offer significant challenges to the bearing and lubrication design.

Additionally the choice of materials for the conical capture surfaces requires close attention, the ability of the faying surfaces to withstand repeated engagements with negligible wear requires careful material selection and lubrication.

This auxiliary bearing system represents a major subassembly in the anomaly control aspects of these flywheel designs. It requires careful design and extensive realistic testing for if it fails to operate after magnetic bearing control is lost, dramatic loss of the high speed rotor will result.

\section{Power Control Electronics}

The function of the power control electronics is to control the rate at which the energy is delivered or extracted from the flywheel. Again this is accomplished using servo control techniques. The energy stored in a flywheel is given by $E=\frac{1}{2} I \omega^{2}$ and the power available is given by $P=T \omega$, where 1 is the flywheel inertia and $\omega$ is the rotational speed of the flywheel. Therefore, controlling the power only requires controlling the motor generator torque to achieve the required power level. A positive torque accelerates the flywheel, storing energy, a negative torque decelerates the flywheel resulting in energy delivery. Figure 7 illustrates this control technique. Power on the spacecraft bus is derived either by extracting energy from the flywheels or by use of solar arrays to convert solar radiation into electrical energy. Exposing the solar arrays to the sun results in an excess of power on the bus. The excess power causes an increase in the bus voltage. The bus voltage is measured and compared to a reference voltage. If the bus voltage is greater than the reference, the voltage regulator issues a positive torque command to absorb power in the flywheel and decrease the bus voltage to its desired value. When the spacecraft enters solar eclipse the bus voltage sags causing the voltage regulator to issue a negative torque command to eliminate the sag. This control technique has two major advantages:

1) the transition from charge to discharge is automatic with no mode switching required

2) a tightly regulated bus voltage is obtained eliminating the need for an additional voltage regulator on the bus.

As shown in Figure 8, controlling the flywheel power requires controlling the motor generator torque. The motor generator of choice for a flywheel energy system is a three phase permanent magnet brushless dc machine. Efficient torque control of this type of machine requires precise motor commutation to minimize non-torque producing currents and low harmonic distortion to minimize harmonic losses. A continuous commutation technique coupled with a high efficiency pulse width modulated inverter is used to achieve these goals.

The torque produced by a three phase brushless dc motor is given by,

$$
T=K_{T}\left[\begin{array}{lll}
i_{a} & i_{b} & i_{c}
\end{array}\right]\left[\begin{array}{c}
\cos (\theta) \\
\cos \left(\theta-2 \frac{\pi}{3}\right) \\
\cos \left(\theta+2 \frac{\pi}{3}\right)
\end{array}\right]
$$

where $i_{a}, i_{b}$ and $i_{c}$ are the motor winding currents, $\theta$ is the angle of the rotor with respect to the magnetic field, and $\mathrm{Kt}$ is the torque constant of the motor. Commutating the motor requires controlling the motor winding currents, $i_{a}$, $i_{b}$, and $i_{c}$, so that the motor torque contains no ac component. A block diagram of the commutation technique is shown in Figure 8. 


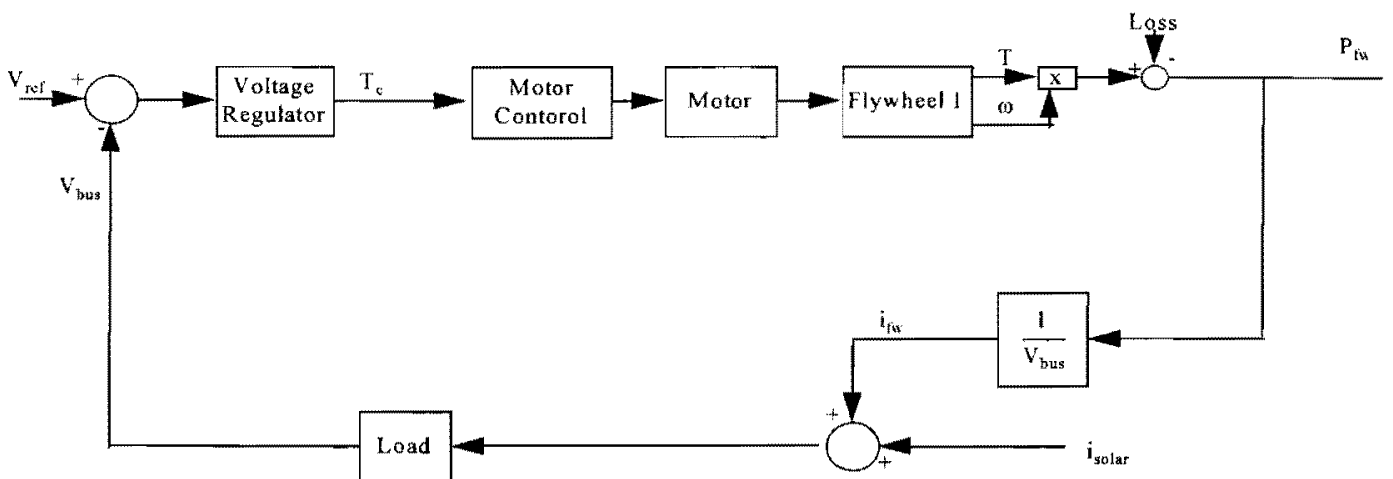

Figure 7: Block Diagram of Power Control System

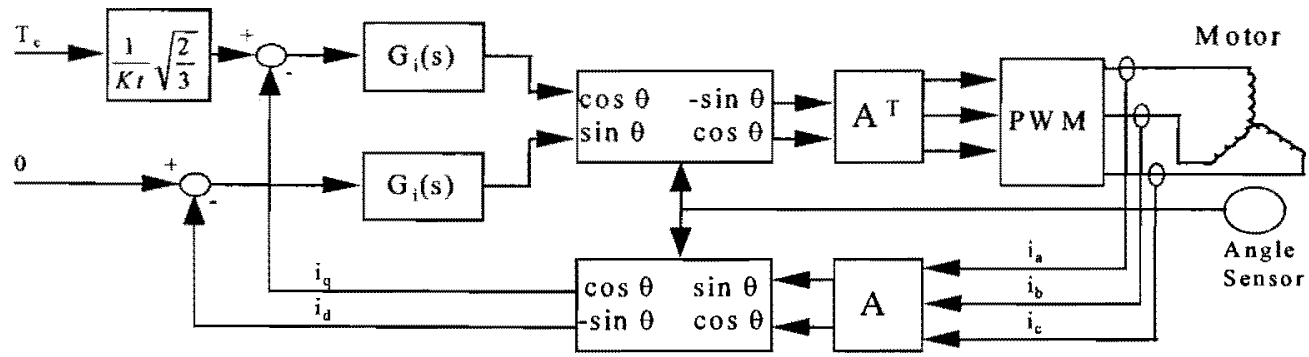

Figure 8: Motor Torque Control

The currents in the motor windings are measured and then transformed into an orthogonal reference frame by a transformation matrix so that

$$
\left[\begin{array}{l}
i_{\alpha} \\
i_{\beta} \\
i_{\gamma}
\end{array}\right]=\sqrt{\frac{2}{3}}\left[\begin{array}{ccc}
1 & -\frac{1}{2} & -\frac{1}{2} \\
0 & \frac{\sqrt{3}}{2} & \frac{\sqrt{3}}{2} \\
\frac{1}{\sqrt{2}} & \frac{1}{\sqrt{2}} & \frac{1}{\sqrt{2}}
\end{array}\right]\left[\begin{array}{l}
i_{a} \\
i_{b} \\
i_{c}
\end{array}\right]
$$

physically, the currents flux producing currents are into a three dimensional space where $i_{\alpha}$ yields the torque producing flux, $i_{\beta}$ is in quadrature to it yielding the field producing flux, and $i_{\gamma}$ is perpendicular to both currents, is zero, and is parallel with the motor shaft. Since $i_{\gamma}$ is zero, only $i_{\alpha}$ and $i_{\beta}$ require control.

Note that $i_{\alpha}$ and $i_{\beta}$ are rotating with respect to the stator. To simplify the control system the currents

SatCon Technology Corporation are transformed into a non-rotating frame by measuring the motor shaft angle and applying the appropriate transformation. At this point a standard current loop is employed to achieve whatever bandwidth response is required. The output of the control laws is then transformed back into the motor winding reference frame to apply the voltage on the windings to achieve the desired currents.

\section{Attitude Control}

Storing energy in the flywheel rotor generates significant angular momentum. When flywheels replace batteries, this momentum is canceled using a counterrotating pair of flywheels. Another approach which results in significant satellite weight savings is to control the angular momentum of an array of energy flywheels to effect attitude control of the spacecraft. Weight and cost savings result from eliminating the reaction wheels or control moment gyroscopes which are typically used as spacecraft attitude actuators.

Selection of a baseline system architecture depends on the specific attitude control and power requirements of the spacecraft. Our approach is to use a spherical momentum envelope with an arrangement of three flywheels mounted so that the momentum 
vectors appear on the faces of a cube, Figure 9. The momentum stored in each flywheel rotor is identical and torque is applied to the spacecraft by rotating the momentum vector in the face of the cube. This is a classical arrangement of momentum devices used in attitude control systems which use control moment gyroscopes (CMGs) as torquers. Energy is stored/ extracted by speeding up/slowing down each flywheel equally.

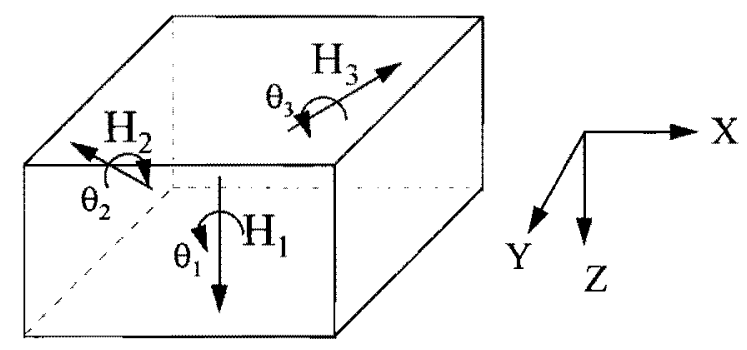

Figure 9. Arrangement of Flywheels for Attitude Control

It is important to recognize that this is not the only arrangement that will work. Examining the system in a general way shows that there are four degrees-offreedom which require control; three axes of rotation and power. Therefore, theoretically, a minimum of two flywheels would be required with control of two wheel speeds and two momentum vector directions. The exact number of units used will depend on redundancy requirements and attitude torque and momentum requirements. In particular the torque requirements will determine if it is feasible to apply torque to the spacecraft directly using the spin motor or if the flywheel needs to be gimbaled. The power required to react torque directly on the spacecraft using the spin motor is $\mathrm{P}=\mathrm{T} \omega$, where $\mathrm{T}$ is the torque in $\mathrm{N}-\mathrm{m}$ and $\omega$ is the wheel speed in rad/s. For high rotational speeds (e.g. $30,000 \mathrm{rpm}$ ) the power required to generate even modest torques becomes prohibitive. For example at a rotor speed of 30,000 rpm the motor/generator shaft power required is 3 $\mathrm{Kw}$ for a torque of $1 \mathrm{~N}-\mathrm{m}$.

Figure 10 is a functional block diagram of a spacecraft using IPACS. To effect attitude control, torque commands are generated by the attitude control computer and fed to the flywheel array. The flywheel converts these commands into magnetic bearing force commands. The magnetic bearings exert a torque on the rotor with the resulting reaction torque being applied to the spacecraft. In reaction to this applied torque the flywheel rotor precesses at an angular rate $\omega_{p}$ determined by the realationship
$T_{o}=\omega_{p} \times H$. The precession motion is compensated for by using the tilt platform to rotate the flywheel housing, thus, assuring the magnetic bearing gaps are not exceeded.

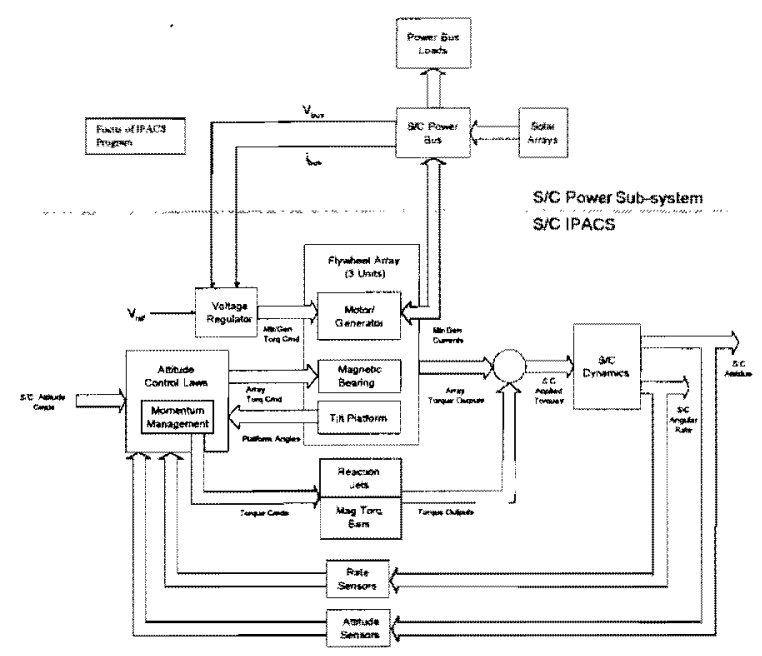

Figure 10. Functional Block Diagram

\section{Operational Considerations}

Spacecraft power and energy storage systems have historically been designed around batteries. For obvious reasons, it is desirable that using a flywheel in lieu of a battery needs to be transparent to the system.

In order for the spacecraft to deploy to its operational configuration, power will be required before the solar arrays become oriented into their power generating attitude. This power need requires either a secondary power source or it implies that the flywheel will be launched rotating at some speed to guarantee that pre-deployment power is available with adequate margin. Similar to batteries, launch site charge capability will be required to ensure that wheel speed is maintained even through a protracted launch hold.

The actual flywheel design will be dictated by its operational requirements. For a GEO application the figure is merit in the energy density at the operational system level, obviously the higher the number the better. Considering launch costs in the range of perhaps $\$ 50 \mathrm{~K} / \mathrm{b}$. the lighter this system, while still providing the necessary energy levels, the more efficient and cost effective the overall system. The ability to provide this weight reduction is one of the main advantages of flywheels. 
In the other extreme, i.e., LEO orbit, different operational conditions give rise to the number of charge/discharge cycles as the figure of merit. Low earth orbit generally means lower launch costs, so overall system weight is not a prime consideration. However, spacecraft at this altitude (less than 400 miles) experience frequent periods of solar eclipse, i.e. in an energy extraction mode (discharge) and solar exposure absorbing energy (charging). LEO vehicles with a ten year life have requirements of 50,000 cycles of charge/discharge. Battery life is severely impacted under these conditions whereas flywheels correctly designed to handle the cyclic stresses and rotor dynamics do not degrade under these stressful conditions.

Using high speed flywheels for energy storage always raises the question of safety, especially in a manned environment such as the Shuttle or Space Station. Flywheels are classified as a Category I hazard. Safety is being handled with a multi-tiered system. The rotor will be designed for infinite life, this will be achieved in a similar manner to the many metal rotors that are now flying. Materials properties will be known, derating factors for stress levels, alternating stress levels and structural safety will be applied. Rotor processing is critical and data on defects, both configuration and size is vital.

Each flywheel assembly will contain an on-board health monitoring system that will monitor rotor imbalance as detected by the magnetic bearings, and/or rim geometry as detected by proximity sensors. Out of tolerance readings from any of these on-board sensors would automatically initiate a built-in shut down system that would rapidly decelerate the flywheel in a manner that is dynamically transparent to the vehicle. Such a system would have restart capability.

\section{Conclusions}

Flywheel Energy Storage Systems offer a viable alternative to the traditional methods of storing energy in the spacecraft environment. Increasing performance requirements from powerful GEO vehicles and the emergence of the LEO vehicles have forced a review of existing systems that are heavy, life limited and generally difficult to handle. Projections for advanced spacecraft indicate that using traditional approaches may consume between 15 to $25 \%$ of the vehicle orbital mass.

SatCon Technology Corporation
Flywheel Energy Storage Systems do offer realistic alternative and considerable efforts are now underway to build and test ground based systems to verify the design approaches. Plans are well advanced to orbit energy storage flywheels first as experiments which if successful will lead directly to flight hardware within the next 5 years.

\section{Acknowledgments}

The engineering staff at SatCon Tucson would like to express our thanks to the management team at SatCon, specifically Dave Eisenhaure and to our many customers for their support and encouragement in the preparation of this paper.

\section{Authors Biographies}

Wilf Robinson - Co-author - General Manager. Mr. Robinson has over 30 years experience in aerospace in England, Canada, and the United States at Spar, Sperry Flight Systems, Ball Aerospace, and Honeywell Space Systems. His primary interest is in mechanisms.

Doug Havenhill - Co-author - Senior Systems Engineer. Mr. Havenhill has over 17 years of spacecraft electromechanical systems experience. In addition to SatCon, he has worked for Sperry Flight Systems, Rocketdyne, and Honeywell Space Systems. His primary expertise is in systems engineering and control systems.

James Hanks - Co-author - Senior Engineer. Mr. Hanks has over 19 years of experience in high speed rotating mechanisms at Westinghouse, Sperry Flight Systems, and Honeywell Space Systems.

Gary Gisler - Co-author - Senior Engineer and Integrated Product Team Leader. Mr. Gisler has over 19 years experience in high speed and spinning aerospace mechanisms at Westinghouse, Sperry Flight Systems, and Honeywell Space Systems.

Len Spina - Co-author - Senior Engineer. Mr. Spina has over 30 years of aerospace power and control electronics experience at Sperry Flight Systems, and Honeywell Space Systems

Dr. Steve Ginter - Co-author - Senior Engineer. Dr. Ginter has over 21 years experience in control systems at Draper Laboratory and Honeywell. His pri- 
mary interest is in spacecraft attitude control and magnetic bearings.

Dr. Sharon Brault - Co-author - Senior Program Manager. Dr. Brault has over 22 years experience in Aerospace at Honeywell and SatCon on electro mechanical systems and the B-52 upgrade program. 